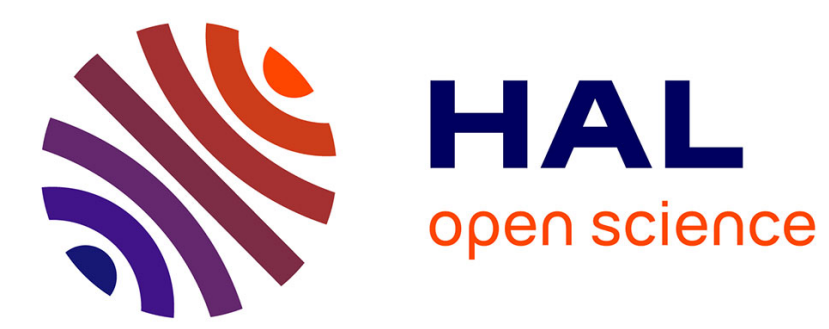

\title{
LE PROBLÈME DU TRAITEMENT DES EAUX RÉSIDUAIRES DE LAITERIE
}

\author{
G. Génin
}

\section{To cite this version:}

G. Génin. LE PROBLÈME DU TRAITEMENT DES EAUX RÉSIDUAIRES DE LAITERIE. Le Lait, 1958, 38 (377), pp.383-389. hal-00928223

\section{HAL Id: hal-00928223 \\ https://hal.science/hal-00928223}

Submitted on 1 Jan 1958

HAL is a multi-disciplinary open access archive for the deposit and dissemination of scientific research documents, whether they are published or not. The documents may come from teaching and research institutions in France or abroad, or from public or private research centers.
L'archive ouverte pluridisciplinaire HAL, est destinée au dépôt et à la diffusion de documents scientifiques de niveau recherche, publiés ou non, émanant des établissements d'enseignement et de recherche français ou étrangers, des laboratoires publics ou privés. 
prendre pour le calcul la dernière valeur obtenue avant l'augmentation de poids.

Dans le cas où cela serait jugé nécessaire, la matière grasse peut être reprise par l'éther de pétrole pour contrôler le résultat de l'analyse.

5. Précision de la méthode.

Matière grasse $\% \pm 0,2 \%$.

\title{
SUPPLEMENT TECHNIQUE \\ LE PROBLÈME DU TRAITEMENT DES EAUX RÉSIDUAIRES DE LAITERIE
}

\author{
par \\ G. GÉNIN \\ Ingénieur E. P. C.
}

Lors d'un récent Congrès de la Pennsylvania Sewage and Industrial Wastes Association, Mr C.L. Siebert du Pennsylvania Department of Health de Harrisburg a présenté une communication sur le problème du traitement des eaux résiduaires de laiterie. Nous reproduisons ci-dessous l'essentiel de cette communication qui a été publiée dans le numéro de septembre 1957 de la revue américaine "Sewage and Industrial Wastes" organe de cette Association. Sans prendre une position définitive, Mr Siebert a passé rapidement en revue les différents procédés dont on dispose pour le traitement des eaux résiduaires de laiterie en insistant plus particulièrement d'ailleurs sur les installations opérant par aération et en rappelant les avantages et inconvénients de ces différents procédés.

\section{Installations fonctionnant par aération.}

Il y a quatre ou cinq ans à peine, un des problèmes qui était le plus discuté parmi les différents procédés dont on disposait à l'époque pour le traitement des eaux résiduaires de laiterie, était de savoir si une installation opérant par simple aération était susceptible de détruire complètement, par oxydation, les résidus solides contenus dans les eaux résiduaires de laiterie.

Cette question s'est trouvée portée à l'ordre du jour quand il y a relativement peu de temps, un certain nombre d'installations de traitement des eaux résiduaires furent mises en service et dans lesquelles aucun des dispositifs classiques de traitement des eaux n'était prévu, sauf une simple aération.

Cette décision fut prise à la suite de recherches de laboratoire qui avaient montré, sur un plan purement expérimental, qu'il est 
possible de réaliser une oxydation complète des boues contenues dans les eaux résiduaires de laiterie en transformant ces boues en eau et en gaz carbonique.

Si ce point avait reçu une réponse positive sur le plan du laboratoire, il restait à établir que ce nouveau procédé de traitement était capable d'être appliqué pratiquement et économiquement dans des installations industrielles.

Aujourd'hui, alors que depuis quelques années déjà un certain nombre d'installations de ce genre, fonctionnant par aération, ont été conçues et réalisées pour le traitement des eaux résiduaires de laiterie, il devient possible de répondre aux objections que l'on avait élevées contre l'emploi de ce procédé.

C'est au cours du Congrès annuel qui s'est tenu en 1956 à University Park (Penn.) de la Pennsylvania Sewage and Industrial Wastes Association que C.L. Siebert a mis au point les résultats déjà acquis et a tiré de l'ensemble des faits aujourd'hui établis les conclusions suivantes.

- Il semble que l'oxydation complète des boues contenues dans les eaux résiduaires de laiterie et leur transformation en eau et en gaz carbonique, si elle est théoriquement possible, se heurte néanmoins à des limitations d'ordre pratique dues au fait que la mise en œuvre de cette méthode conduit fréquemment à des périodes de marche au cours desquelles l'installation se trouve amenée au-delà de ses limites normales d'emploi.

- D'autre part, ce procédé d'aération des eaux résiduaires conduit à diriger dans les eaux une quantité d'air importante pour assurer une oxydation complète des boues et cela entraîne des dépenses assez élevées d'énergie. Pour que l'opération reste économique, il semble que dans l'état actuel des choses, elle doit être limitée aux eaux provenant d'installations d'importance réduite.

Fonctionnement continu ou discontinu de l'installation.

Actuellement, des discussions qui n'ont pas encore conduit à une conclusion définitive se sont élevées sur le fait de savoir si des installations opérant par aération et travaillant d'une façon continue sont techniquement supérieures à des installations fonctionnant au contraire d'une façon discontinue dans lesquelles on opère sur des lots successifs d'eaux résiduaires à traiter. Il est certain que si une réponse pouvait être trouvée à ces questions, cela présenterait un grand intérêt. Or, jusqu'à ce jour, les ingénieurs du Pennsylvania Department of Health auquel appartient l'auteur de la communication dont nous reproduisons une analyse très complète, n'ont pas été capables de se former une opinion définitive sur ce point. La raison principale de cette incertitude réside dans le fait qu'il n'existe 
pas en Pennsylvanie d'installation industrielle sur laquelle on ait pu faire des essais suivis et opérant sur des lots successifs d'eaux à traiter dans des conditions convenables.

Il semble cependant que le procédé dans lequel on travaille d'une façon discontinue sous sa forme actuelle ne permet pas de rejeter des eaux régulièrement épurées et pour cette raison, il présente de sérieux inconvénients.

\section{Dispositif pour la digestion des boues.}

Dans les installations de traitement des eaux résiduaires de laiterie qui avaient été créées il y a huit à dix ans pour les établissements industriels importants, tels que celles des National Dairies, il avait été prévu dans l'installation un réservoir spécial où devait s'effectuer la digestion des boues. Or, il est apparu que plusieurs de ces installations pouvaient fonctionner pendant un an ou plus sans qu'elles aient à être périodiquement nettoyées, afin que les boues formées soient recueillies et traitées séparément dans les appareils de digestion. Il en résulte que ces dispositifs particuliers ont été supprimés dans certaines installations d'apparition plus récente. Mr C.L. Siebert estime cependant qu'il y aura toujours intérêt dans une installation de traitement des eaux résiduaires à prévoir une installation de digestion, même si elle est de faible capacité, ayant par exemple un volume de 1.500 à 3.000 litres, pour recevoir les boues en excès qui peuvent se former exceptionnellement à la suite d'un accident survenu dans la fabrication.

Il est probable qu'un des principaux reproches que l'on ait attribué au mauvais fonctionnement des installations d'aération fonctionnant d'une façon discontinue, est le manque d'oxygène nécessaire au traitement des boues dans des cuves de décantation. Assez fréquemment en effet les quantités de boues qui se trouvent envoyées dans ces cuves de décantation sont mal réglées et insuffisantes, les opérateurs estimant que pour obtenir une bonne clarification c'est-à-dire une décantation convenable, le liquide contenu dans la cuve doit être maintenu au repos autant que possible. Il peut en résulter une transformation biologique des organes vivants' dans ces cuves de décantation. Ces organes en effet sont constitués de protoplasme et si un manque d'oxygène se produit, il faut immédiatement retourner les boues dans une cuve où ces organismes vivants trouveront suffisamment d'oxygène pour pouvoir vivre.

Rejet de boues ayant subi une digestion partielle.

Il y a un certain temps; S.S. Hoove f de l'Agricultural Research Service du Ministère américain de l'Agriculture avait pensé qu'il 
serait possible d'obtenir par aération une boue dans laquelle l'oxydation biologique ne serait pas menée complètement à son achèvement, mais arrêtée à un point où la boue pourrait être considérée comme stabilisée et pourrait être à ce moment rejetée dans un cours d'eau sans entraîner une consommation excessive de l'oxygène contenu dans ce cours d'eau.

C'est à la suite de cette hypothèse que R.R. Kountz a effectué un essai sur plan industriel, mais on a constaté que la boue considérée comme stabilisée à la sortie de l'installation de traitement s'accumulait en fait dans le cours d'eau et entraînait d'importants dégâts pour la vie aquatique. Cette constatation confirmait d'ailleurs des observations antérieures faites au cours de travaux entrepris par C.L. Siebert.

Pour un observateur superficiel, le déplacement des eaux dans un cours d'eau est à priori beaucoup plus rapide que celui qu'on observe dans une cuve de décantation. En fait, l'expérience a montré que même dans des cours d'eau relativement peu importants, il existe en certains points des plages où les eaux sont calmes et où des phénomènes de décantation peuvent se produire au moins aussi facilement que dans une cuve de décantation. Il en résulte en ces endroits une accumulation de matières en suspension. Malgré que certains petits cours d'eau semblent pour un observateur bénéficier d'un courant plus rapide que celui de fleuves plus importants, en fait la vitesse moyenne des eaux est souvent plus faible et l'existence de zones calmes est très fréquente. Les boues déposées en ces endroits forment une couche de matières qui se concentrent dansle lit du cours d'eau, les organismes vivants ne trouvent plus suffisamment d'oxygène, ils meurent et subissent une décomposition anaérobique. Il est donc indispensable que les eaux traitées rejetées contiennent le moins possible de boues. En fait, il semble que les autorités s'opposeront toujours au rejet dans les cours d'eau de boues apparemment stabilisées, mais dont la présence peut conduire à de graves accidents.

\section{Dispositifs d'aération.}

D'intéressants perfectionnements ont été apportés récemment aux dispositifs d'aération. Les diffuseurs comportant des orifices extrêmement réduits et dans lesquels aucune pièce mobile n'existe ont cependant laissé plus ou moins à désirer jusqu'ici car il est presque impossible de les maintenir en bon état. Le fait que les eaux résiduaires de laiterie forment facilement des dépôts est partieulièrement préjudiciable à ce genre de dispositif et il est à prévoir que cet inconvénient subsistera dans tous les types de diffuseurs à très faibles orifices. 
On a cependant cherché à remédier à cet inconvénient par différents artifices, comme par exemple l'injection de vapeur dans les conduites de distribution d'air comprimé. Dans certains cas, cette solution s'est révélée favorable, mais dans d'autres cas inefficace Il ne semble pas qu'on dispose aujourd'hui d'un procédé universel permettant de déboucher les orifices obstrués.

C'est la raison pour laquelle dans le plus grand nombre des installations actuelles, on tend à utiliser les dispositifs d'aération ayant des orifices d'environ 3 millimètres de diamètre. Les dispositifs d'aération sont constitués par des tuyauteries comportant des orifices de cette dimension, et s'il est certain que le taux d'utilisation de l'air se trouve réduit, il n'en reste pas moins vrai que l'efficacité de ces installations reste raisonnable et surtout les dépenses d'entretien sont réduites au minimum, car le colmatage des orifices est beaucoup moins fréquent.

On a également utilisé pour réaliser l'aération des boues des injecteurs du type Penberthy. Ces dispositifs se sont révélés d'un fonctionnement sûr et ils sont efficaces au point de vue du coefficient d'utilisation de l'air, mais ils conduisent à d'importantes dépenses d'énergie de pompage qui peuvent devenir excessives dans certaines grandes installation. Toutefois, C. L. Simbert a signalé dans sa communication qu'il n'avait jamais été constaté de colmatage des orifices dans des dispositifs de ce genre.

\section{Filtres à ruissellement.}

Les filtres à ruissellement continuent à être utilisés pour le traitement des eaux résiduaires de laiterie. Dans ce cas particulier, le fonctionnement de ces dispositifs ne se distingue en rien de celui des filtres du même type employé pour le traitement d'eaux résiduaires de toutes sortes. Leur principal inconvénient est leur prix relativement élevé et la raison principale pour laquelle, en particulier dans les installations de moyenne importance, on a cherché à adopter des procédés de traitement par aération, a été l'espoir de réaliser des économies dans la construction des installations.

Une installation par aération présente d'autre part un autre inconvénient non négligeable. En effet, en hiver, par temps froid, il se produit sur un filtre à ruissellement une diminution importante de la température des eaux à traiter, beaucoup plus élevée que celle qu'on constate dans une cuve contenant un dispositif d'aération. Il peut en résulter que la température de l'eau s'abaisse à une valeur insuffisante pour que les phénomènes biologiques puissent s'effectuer.

\section{L'utilisation des eaux résiduaires pour l'irrigation.}

Dans sa communication, C.L. Siebert a indiqué qu'il existe en 
Pennsylvanie une usine où les eaux résiduaires sont utilisées pour l'irrigation de certaines cultures. Dans cette usine, les eaux résiduaires sont recueillies dans une soute, puis périodiquement transvasées au moyen de camions-citernes dans les champs des villages avoisinants où ces eaux résiduaires se trouvent pulvérisées à la surface du sol.

Il est inutile de dire que les terrains où se fait cette irrigation doivent être obligatoirement éloignés de tout endroit habité et pour des raisons d'hygiène éloignés également de la laiterie. Aucune indication n'a été communiquée sur les dépenses qu'entraîne ce mode d'utilisation des eaux résiduaires ; il est possible qu'il présente un certain intérêt dans des cas bien déterminés.

On a également signalé dans certains endroits l'utilisation des eaux résiduaires pour l'irrigation, ces eaux étant conduites sur les lieux d'utilisation au moyen de tuyauteries aux extrémités desquelles se trouvent des dispositifs de pulvérisation.

Il est possible d'ailleurs de faire un rapprochement entre ce mode de traitement des eaux et le traitement sur filtre à ruissellement, car on peut dire que la terre et la végétation agissent elles aussi comme un filtre à ruissellement sur lequel les eaux se trouvent réparties en couches très minces. Les bactéries qui existent dans le sol sont du même type que celles qu'on trouve dans un filtre à ruissellement ou dans une installation d'épuration au moyen de boues activées et agissent donc de la même façon.

\section{Le problème des eaux de lavage fournies par la ferme.}

Aux Etats-Unis tout au moins, on tend de plus en plus, pour réduire les frais de ramassage du lait à assurer le transport de celui-ci dans des camions-citernes et cette tendance tendra sans aucun doute à se développer. Il en résulte que le fermier doit procéder périodiquement au lavage des réservoirs et d'une façon générale de tout le matériel ayant contenu du lait et il en résulte que les points de rejet des eaux de lavage, au lieu de se trouver concentrés aux points de ramassage ou dans les laiteries, se trouvent dispersés sur tout le territoire.

C'est cette dispersion qui semble à priori favoriser le problème, car on peut penser qu'il est moins dangereux au point de vue pollution d'un cours d'eau ou de ses affluents de rejeter des eaux résiduaires en de multiples points, plutôt que de concentrer ces eaux en un seul point voisin de l'établissement industriel. Cependant, ce fait n'est pas rigoureusement établi et rien n'indique que la multiplication des points de pollution ne conduise pas les autorités à prendre à l'avenir des mesures s'opposant au rejet des eaux résiduaires provenant des fermes laitières. La question se posera 
donc, peut être plus tôt qu'on ne le pense, d'imposer aux fermiers des installations d'épuration des eaux.

En résumé, il semble done que la situation concernant le choix définitif des installations d'épuration des eaux résiduaires de laiterie ne soit pas encore totalement clarifiée. Les différents dispositifs dont on dispose aujourd'hui présentent chacun leurs avantages et leur inconvénients. Aux yeux des spécialistes américains qui prévoient une concentration des établissements industriels et des centres de ramassage, il semble que le problème actuel soit surtout celui de disposer d'installations de traitement des eaux résiduaires aptes à supporter des surcharges passagères assez importantes pour répondre aux variations de la production saisonnières du lait et surtout des installations susceptibles de permettre une augmentation progressive de leur capacité de traitement ne conduisant pas à des transformations profondes et pouvant au contraire suivre régulièrement les besoins des centres de ramassage ou des laiteries au fur et à mesure du développement de l'activité de ces établissements.

\section{Bulletin analytique}

\section{Revues}

\section{Colles et peintures}

\section{Liss (Z.). - L'influence des sels, des pigments et des agents de conservation sur la stabilité des vernis pigmentés à base de caséine pour cuir. Kozarstvi, 1955, t. V, p. 65-6.}

On a apprécié la décomposition des vernis pigmentés à base de caséine en procédant à une mesure des variations des viscosités de ce produit, La vitesse de décomposition dépend de la nature du pigment et diminue dans l'ordre Ecarlate Versal RNL, jaune Versal 5G, bleu de phtalocyanine, noir de carbone, bordeaux graphtol HB, rouge d'oxyde de fer (le produit ne se décomposa pas après 8 mois). Les liants sans pigments ne se décomposent pas en 6 mois. La décomposition est catalysée par les pigments ou par les sels solubles dans l'eau qu'ils contiennent. C'est ainsi par exemple que les extraits aqueux d'écarlate et de jaune Versal renferment des ions fer, calcium, chlore, $\mathrm{SO}_{4}$ et $\mathrm{NO}_{3}$.

Une solution à $15 \%$ de caséine contenant $1 \%$ de parachlorométacrésol subit une coagulation partielle immédiate lorsqu'on la traite avec $0,25 \%$ de chlorure ferrique, de sulfate ferreux, de sulfate ferrique, de chlorure de calcium, ou de nitrate de calcium et au bout de 21 jours à $40^{\circ}$, la viscosité s'abaisse à celle de l'eau. Les pigments à base de caésine peuvent avoir une conservation assurée en leur ajoutant une plus grande quantité de parachlorométacrésol et il faut une plus forte proportion de cet agent pour des solutions 